\title{
Atypical perinuclear antineutrophil cytoplasmic antibodies in patients with Crohn's disease
}

\author{
Hugh J Freeman MD
}

HJ Freeman. Atypical perinuclear antineutrophil cytoplasmic antibodies in patients with Crohn's disease. Can J Gastroenterol 1997;11(8):689-693. Atypical perinuclear antineutrophil cytoplasmic antibodies ( $\mathrm{p}$-ANCA) occur in most patients with ulcerative colitis but only in a minority of those with Crohn's disease. In a recent study from the United States, this serological marker was reported to be present in $100 \%$ of Crohn's disease patients with a clinical phenotype of 'left-sided ulcerative colitis' but was not present in patients with 'isolated' small bowel disease. In a previously reported survey from the author's hospital, the pANCA status of 247 consecutive patients with Crohn's disease was evaluated, and, of these, 33 Crohn's disease patients were seropositive, including $18(13.0 \%)$ with combined small and large bowel disease, 11 (19.6\%) with 'isolated' colorectal disease, and four ( $4.6 \%)$ with 'isolated' small bowel but no detectable colorectal disease. To further evaluate and verify the significance of atypical p-ANCA in these 33 patients, clinical, radiological, endoscopic and histological features were examined. This study confirms that an 'ulcerative colitis-like' clinical phenotype may be seen in most, but not all, serologically positive Crohn's disease patients. Moreover, 'isolated' small bowel disease in the absence of colorectal disease occurs. Detection of atypical p-ANCA in Crohn's disease with different clinical and pathological features provides serological evidence that Crohn's disease is a very heterogeneous disorder.

Key Words: Crohn's disease, Inflammatory bowel disease, Neutrophil cytoplasmic auto-antibodies, Perinuclear antineutrophil cytoplasmic antibodies

$\mathrm{P}$ revious studies, including a prospective evaluation of 500 consecutive patients with inflammatory bowel disease from my clinical practice, revealed that atypical perinuclear antineutrophil cytoplasmic antibodies (p-ANCA)

\section{Anticorps cytoplasmiques antineutrophiles atypiques périnucléaires chez des patients atteints d'une maladie de Crohn}

RÉSUMÉ : Les anticorps cytoplasmiques antineutrophiles atypiques périnucléaires ( $\mathrm{p}$-ANCA) s'observent chez la plupart des patients qui souffrent de colite ulcéreuse mais chez une minorité de ceux qui souffrent de la maladie de Crohn. Dans le cadre d'une récente étude américaine, ce marqueur sérologique a été signalé dans $100 \%$ des cas de maladie de Crohn en présence d'un phénotype clinique de colite ulcéreuse du côté gauche mais s'est révélé absent chez les patients qui souffraient de maladie du grêle isolée. Dans le cadre d'une enquête qui a déjà fait l'objet d'un rapport à notre hôpital, le statut des p-ANCA chez 247 patients consécutifs atteints de maladie de Crohn a été évalué et, parmi eux, 33 patients atteints de maladie de Crohn étaient séropositifs, y compris 18 (13\%) qui souffrant de maladie combinée du petit et du gros intestin, $11(19,6 \%)$ qui souffraient de maladie recto-colique isolée et $4(4,6 \%)$ qui souffraient d'une atteinte isolée du grêle sans maladie recto-colique décelable. Pour approfondir l'évaluation et vérifier la portée des p-ANCA atypiques chez ces 33 patients, les caractéristiques cliniques, radiologiques, endoscopiques et histologiques ont été examinées. Cette étude confirme qu'un phénotype clinique de pseudo-colite ulcéreuse peut s'observer chez la plupart mais non chez tous les patients atteints de maladie de Crohn sérologiquement positifs. De plus, la maladie du grêle isolée en l'absence de maladie recto-colique s'observe. Le dépistage des pANCA atypiques dans la maladie de Crohn, avec différentes caractéristiques cliniques et pathologiques, offre les preuves sérologiques que la maladie de Crohn est très hétérogène.

Department of Medicine (Gastroenterology), University of British Columbia, Vancouver, British Columbia

Correspondence and reprints: Dr Hugh Freeman, ACU F-137, Gastroenterology, Vancouver Hospital (UBC site), 2211 Wesbrook Mall,

Vancouver, British Columbia V6T 1W5. Telephone 604-822-7216, fax 604-822-7236

Received for publication May 9, 1997. Accepted August 20, 1997 
with this seromarker (4). More specifically, $100 \%$ of all seropositive Crohn's disease patients in that report had a distinctive phenotype with symptoms ascribed to left-sided colonic disease (ie, rectal bleeding and urgency), as well as endoscopic and/or histopathological evidence of left-sided colitis. Moreover, all seropositive patients had colonic disease without 'isolated' small intestinal disease. If these results could be confirmed, then a positive serological test in a Crohn's disease patient would have potentially important clinical, especially therapeutic, implications. As a result of that report (4), the clinical and pathological data in patients with Crohn's disease from our previous study (3) were further evaluated. Specifically, in 247 consecutive patients with Crohn's disease, the clinical profile and location of disease in each seropositive patient were determined with the use of radiological, endoscopic and histopathological methods. Although left-sided colonic disease was present in some, but not all, seropositive patients, significant differences were also evident, including detection of four patients with Crohn's disease involving the small intestine without any evidence of colonic disease.

\section{PATIENTS AND METHODS}

Patient groups and laboratory studies: Patients had an established diagnosis of Crohn's disease in the small and/or large intestine based on clinical, radiological, endoscopic and histological criteria as well as negative microbiological studies (3). Further characterization of patients with features of an ulcerative colitis or ulcerative colitis-like inflammatory process was based on the specific clinical criteria defined in a prior report by Vasiliauskas et al (4). Briefly, these criteria include rectal bleeding with or without mucous discharge, urgency, tenesmus and treatment with topical therapies. Radiological, endoscopic and histological evaluations were also used to define the site, location and extent of disease.

In the present study, however, pathological evaluation also included histological definition of the presence or absence of either granulomas or multinucleated giant cells in endoscopic biopsy and/or surgical specimens for all 33 Crohn's disease patients. For each patient, hospital pathological reports were reviewed and a formal histological evaluation of available serially sectioned materials for each patient was done.

ANCA immunofluorescence: The previously reported method for immunofluorescence detection of atypical pANCA was used $(1,3)$. Specifically, indirect immunofluorescence for cytoplasmic ANCA, p-ANCA and atypical pANCA was done with a fluorescent antibody detection method (Inova Diagnostics Inc, California) using slides with an adherent layer of cultured human neutrophils fixed with either ethanol or formalin. This method is identical to methods used in studies of patients with Crohn's disease $(3,4)$.

\section{RESULTS}

Patients with Crohn's disease: Thirty-three seropositive patients were studied from 247 consecutive patients with Crohn's disease who were evaluated (Table 1). There were 18 females (55\%) and 15 males (45\%), ranging in age from 14 to 43 years at the time of diagnosis of Crohn's disease. This compared with the overall group with Crohn's disease consisting of 146 females (59\%) and 101 males (41\%), ranging in age from nine to 81 years at the time of diagnosis, and with the seronegative patients with Crohn's disease consisting of 128 females (59\%) and 86 males (41\%). The mean duration of follow-up by the author (or the patient's prior gastroenterologist/pediatric gastroenterologist) after initial diagnosis for patients with seropositive Crohn's disease was 10.5 years, ranging from one to 42 years. Diagnosis of Crohn's disease was established by another physician for five of these $33 \mathrm{pa}$ tients (all before 1981) - diagnosis was confirmed in each of these cases.

Features of Crohn's disease: At least seven of the 33 seropositive patients had fistulizing disease, reflected in a visible enterocutaneous or enterovaginal fistula requiring surgical treatment. Another 12 of these 33 had perianal disease, usually a localized abscess, treated with one or more antimicrobial agents (including metronidazole, ciprofloxacin or both) as well as surgical treatment. Of these, 10 had colonic or ileocolonic involvement documented, while two had 'isolated' perianal disease with no evident colonic disease. The 33 seropositive patients received an oral 5-aminosalicylatecontaining medication, prednisone or, usually, both medications. Two patients were treated with oral controlled ileal release capsules of budesonide. Thirteen patients had at least one small bowel resection, usually with a partial colectomy, and five had a complete proctocolectomy. One patient with a prior proctocolectomy also had primary sclerosing cholangitis complicated by clinical evidence of portal hypertension with stomal varices - he had a liver transplant following the onset of liver failure (5) and currently receives cyclosporine.

Assessment: According to the clinical criteria of Vasiliauskas et al (4) to define 'ulcerative colitis-like' disease, 19 of 33 seropositive patients had symptoms that suggested left-sided colonic disease. Of these, only 10 had radiological, endoscopic and histopathological definition of disease localized in the colon alone. The other nine patients with clinical features of ulcerative colitis-like disease also had involvement of the distal small intestine as well as the colon. No patient with these ulcerative colitis-like clinical criteria had disease isolated in the small intestine. In addition, nine patients also had perianal disease, and five had clinically evident fistulous disease.

Histological evaluation for granulomas: Twenty-eight patients $(85 \%)$ had granulomas detected in at least one site from surgically resected or endoscopic biopsy material. In 17 of the 19 patients ( $89 \%$ ) with ulcerative colitis-like Crohn's disease, granulomas (or multinucleated giant cells) were histologically confirmed from biopsy or surgical materials from the colon. Of the 14 patients without clinical criteria of ulcerative colitis-like disease, none had isolated colorectal disease, four had small bowel disease without detectable colorectal disease, and the remaining 10 had ileocolonic disease. Of these 14 patients, three had perianal disease and two 
TABLE 1

Crohn's disease patients positive for antineutrophil cytoplasmic antibodies

\begin{tabular}{|c|c|c|c|c|}
\hline Age/sex & $\begin{array}{l}\text { Year of } \\
\text { diagnosis }\end{array}$ & $\begin{array}{l}\text { Ulcerative } \\
\text { colitis-like }\end{array}$ & Disease site & Treatment \\
\hline 18/female & 1986 & Yes & Ileum*, colon*, PA & 5-ASA, prednisone, imuran \\
\hline 17/female & 1973 & Yes & lleum $^{*}$, colon $^{*}$, PA & $\begin{array}{l}\text { 5-ASA, prednisone, SBR and } \mathrm{PaC} \text {, stricturoplasty, } \\
\text { proctocolectomy }\end{array}$ \\
\hline 23/female & 1987 & No & $\begin{array}{l}\text { Stomach*, duodenum }{ }^{*} \\
\text { ileum*, PA }\end{array}$ & 5-ASA, prednisone, metronidazole, $\mathrm{SBR}$ and $\mathrm{PaC}$ \\
\hline $14 /$ male & 1964 & Yes & lleum*, colon*, PSC, varices & $\begin{array}{l}\text { 5-ASA, prednisone, SBR, proctocolectomy, liver } \\
\text { transplant, cyclosporine }\end{array}$ \\
\hline 34/female & 1987 & No & Ileum, colon & 5-ASA, prednisone, SBR, $\mathrm{PaC}$ \\
\hline $18 /$ male & 1982 & Yes & lleum $^{*}$, colon $^{*}$, PA & 5-ASA, prednisone, 6-mercaptopurine \\
\hline $21 /$ male & 1993 & Yes & Colon* & 5-ASA, prednisone \\
\hline 24/female & 1985 & No & $\begin{array}{l}\text { Stomach*, duodenum }{ }^{\star} \text {, } \\
\text { ileum } \text { colon }^{\star}\end{array}$ & 5-ASA, prednisone, omeprazole \\
\hline 42/female & 1994 & Yes & Colon* & 5-ASA \\
\hline $23 /$ male & 1995 & No & Ileum* ${ }^{*}$ colon* & 5-ASA, prednisone, SBR, $\mathrm{PaC}$ \\
\hline $15 /$ male & 1988 & No & lleum*, PA, EC-F & 5-ASA, prednisone, SBR, $\mathrm{PaC}$ \\
\hline 23/female & 1989 & No & Ileum, colon & 5-ASA, prednisone, budesonide \\
\hline $21 /$ female & 1982 & No & lleum* ${ }^{*}$ colon* & 5-ASA, prednisone, SBR, $\mathrm{PaC}$ \\
\hline $27 /$ male & 1967 & Yes & Colon*, PA, EC-F & Prednisone, proctocolectomy \\
\hline $17 /$ male & 1993 & No & Ileum, colon & 5-ASA, prednisone \\
\hline $31 /$ male & 1989 & Yes & Colon*, PA & 5-ASA, metronidazole \\
\hline $33 /$ male & 1990 & Yes & lleum ${ }^{*}$, colon* & 5-ASA, prednisone \\
\hline $24 /$ male & 1977 & No & $\begin{array}{l}\text { Stomach*, duodenum*, } \\
\text { ileum, colon }\end{array}$ & $\begin{array}{l}\text { 5-ASA, prednisone, SBR and } \mathrm{PaC} \text {, and vagotomy } \\
\text { and pyloroplasty }\end{array}$ \\
\hline $21 /$ female & 1990 & Yes & Ileum, colon & 5-ASA, prednisone, budesonide, SBR, $\mathrm{PaC}$ \\
\hline 22/female & 1994 & Yes & Colon*, PA, RV-F & $\begin{array}{l}\text { 5-ASA, prednisone, metronidazole/ciprofloxacin, } \\
\text { proctocolectomy }\end{array}$ \\
\hline $36 /$ female & 1987 & No & lleum ${ }^{*}$, colon*, PA, RV-F & 5-ASA, lansoprazole, SBR, $\mathrm{PaC}$ \\
\hline 17/female & 1984 & Yes & Colon*, EC-F & $\begin{array}{l}\text { 5-ASA, prednisone, metronidazole/ciprofloxacin, } \\
\text { proctocolectomy }\end{array}$ \\
\hline 39/female & 1994 & Yes & Colon, PA, RV-F & 5-ASA \\
\hline $21 /$ female & 1983 & Yes & Ileum ${ }^{*}$, colon*, PA, EC-F & 5-ASA, prednisone, proctocolectomy \\
\hline $16 /$ male & 1987 & No & lleum*, colon* & 5-ASA, prednisone \\
\hline 28/female & 1985 & Yes & $\begin{array}{l}\text { lleum }{ }^{\star}, \text { colon}^{\star}, \text { PA, EC-F, } \\
\text { ileosigmoid fistula }\end{array}$ & 5-ASA, prednisone, SBR, $\mathrm{PaC}$ \\
\hline 32/female & 1955 & No & lleum* & Prednisone, SBR, PaC \\
\hline $43 /$ male & 1992 & Yes & Colon* & 5-ASA, prednisone \\
\hline $21 /$ male & 1995 & Yes & Colon* & 5-ASA \\
\hline $22 /$ male & 1982 & No & lleum* & 5-ASA, prednisone \\
\hline $25 /$ female & 1983 & Yes & Ileum, colon* & 5-ASA, prednisone, SBR, $\mathrm{PaC}$ \\
\hline $29 /$ male & 1995 & No & Ileum, colon* & 5-ASA \\
\hline 19/female & 1986 & Yes & Colon* & 5-ASA, prednisone, $\mathrm{PaC}$ \\
\hline
\end{tabular}

*Granuloma or multinucleated giant cells. 5-ASA 5-aminosalicylate; EC-F Enterocutaneous fistula; PA Perianal involvement; PaC Partial colectomy; PSC Primary sclerosing cholangitis; RV-F Rectovaginal fistula; SBR Small bowel resection

had clinically evident fistulous disease. Of these 14 patients, 11 had histological confirmation of granulomas (or multinucleated giant cells) in biopsy or surgical materials from the gastrointestinal tract. Of the three seropositive Crohn's disease patients with gastric involvement (reflected by the pres- ence of granulomas in endoscopic gastric mucosal biopsies) (6), none had ulcerative colitis-like clinical features.

Effects of therapies in seropositive Crohn's disease: Seropositive patients with Crohn's disease received a variety of medications, including 5-aminosalicylate-containing medi- 
cations and corticosteroids, as well as surgical therapies, including partial small and large intestinal resections. In spite of a total proctocolectomy, all four patients with Crohn's disease in this investigation, as in ulcerative colitis patients, were seropositive (5). Another patient also had a liver transplant for sclerosing cholangitis.

Analysis of disease localization in seropositive Crohn's disease: Disease localization was defined by radiographical, endoscopic and histopathological methods in all 33 seropositive Crohn's disease patients. There were 19 with ileocolonic disease, 10 with colorectal disease and four with small intestinal but not colorectal disease. Of these, two patients with ileocolonic disease and one with disease localized in the distal ileum also had gastric and duodenal disease. Granulomas were detected in 14 of 19 seropositive patients with ileocolonic disease, eight of 10 with colorectal disease, and four of four with ileal disease but no colorectal disease.

Cases with small bowel but not large bowel involvement: Case 1: A 23-year-old female was evaluated in January 1987 for abdominal pain. Upper gastrointestinal endoscopy revealed multiple gastric erosions and granularity of the duodenal bulbar mucosa.

Biopsies of the duodenum, gastric antrum and gastric body revealed a focally active mucosal inflammatory process with multiple noncaseating mucosal granulomata (6). Helicobacter pylori was not seen. She was treated with oral ranitidine, and her symptoms resolved. Because of diarrhea and weight loss, she was evaluated in June 1988. Barium radiographs of the upper gastrointestinal tract revealed terminal ileal stenosis with ulceration. Bloodwork including a hemogram, sedimentation rate, liver chemistry tests, iron and iron binding capacity, and serum proteins including albumin were normal. Fecal cultures and parasite studies were negative. She was treated with ranitidine and Asacol (Proctor and Gamble Pharmaceuticals). Flexible sigmoidoscopy and a rectal biopsy were normal. A barium enema showed a normal colon. She remained on Asacol until July 1994. At that time, abdominal pain recurred with weight loss. Gastroscopy and colonoscopy were normal, and multiple biopsies of the duodenum and colon were normal. Gastric biopsies, however, showed only focal gastritis with no granulomatous inflammation or $\mathrm{H}$ pylori. Barium studies revealed a $20 \mathrm{~cm}$ segment of distal ileal disease with multiple isolated areas of stenosis. Surgical resection of this ileal segment was done in August 1995.

Pathological evaluation revealed transmural acute and chronic inflammation in the distal ileum with ulceration and granuloma formation. In March 1997 she presented with features of a small bowel obstruction that resolved with intravenous fluids and nasogastric suction. Endoscopic and radiological studies of the upper and lower gastrointestinal tract showed recurrent 'neoterminal' ileal disease, estimated to be about a $30 \mathrm{~cm}$ length of the distal small intestine with ulceration. No colonic disease was visualized, and colonic mucosal biopsies were normal. She was treated with oral Pentasa (Hoechst Marion Roussel), and her symptoms have resolved.
Case 2: A 15-year-old male was evaluated in February 1988 with a history of two months of severe but intermittent abdominal pain. At a rural community hospital two weeks earlier, a tentative diagnosis of appendicitis had been made, and a laparotomy done. Two 'skip' lesions involving the distal ileum, typical of Crohn's disease, were seen. An appendectomy showed granulomatous appendicitis, and the patient's postoperative course was uncomplicated. Stains and cultures of the resected specimen were negative, including acid-fast (Ziehl-Neilson) stains and cultures for Mycobacterium tuberculosis. Serological studies and fecal bacterial cultures, done to exclude other causes for granulomatous appendicitis (ie, Yersinia species), were negative. Barium radiological studies of the upper gastrointestinal tract showed stenosis and ulceration of the distal ileum. Pain recurred with fever, and an abdominal ultrasound with computerized tomographic scans revealed thickened bowel with an inflammatory mass. Endoscopic evaluation (colonoscopy and ileoscopy) showed ileal ulceration, and ileal mucosal biopsies confirmed inflammation. The colon was normal, and colonic biopsies were normal. Another $32 \mathrm{~cm}$ ileal segment was resected. Focal transmural ileal inflammation with granulomas in the intestinal wall and mesenteric lymph nodes were present. Since this resection, there have been no symptoms. In May 1994. Asacol was changed to Pentasa, and in June 1995, laboratory studies (hemogram, serum iron studies, sedimentation rate and serum proteins including serum albumin) were normal.

Case 3: A 35-year-old Australian male was diagnosed with Crohn's disease of the terminal ileum in 1982. The resected ileal specimen showed granulomas. At that time he was treated with a course of prednisone followed by sulphasalazine. He remained asymptomatic until May 1995. While touring in British Columbia he developed fever, abdominal pain and diarrhea. Except for a hemoglobin of $128 \mathrm{~g} / \mathrm{L}$, laboratory studies were normal. Flexible sigmoidoscopy and rectal mucosal biopsy were normal. Abdominal ultrasound was normal. Barium radiological studies of the upper and lower gastrointestinal tracts showed a narrowed, deformed, cobblestoned terminal ileum. Treatment with added corticosteroids led to resolution of symptoms. In September 1995 the patient returned to Australia, completely asymptomatic on sulphasalazine alone.

Case 4: A 60-year-old female was first evaluated for abdominal pain in September 1987. In 1955, Crohn's disease of the distal ileum was diagnosed. In 1965, an ileal resection was done at the Beth Israel Hospital, Boston, Massachusetts. The resected ileum had deep fissures and ulceration with granulomas. Physical examination and laboratory tests, including urinalysis, were normal. Abdominal ultrasound was normal. Endoscopic and radiological studies of her upper and lower gastrointestinal tract were normal, as were endoscopic biopsies of her stomach, and small and large intestines. Two subsequent evaluations elsewhere, including endoscopic and radiological evaluations of her upper and lower gastrointestinal tracts, for abdominal pain in July 1992 and August 1993 did not establish a cause of her pain. On both occasions, her 
pain resolved spontaneously with no medications. In January 1995, a urological evaluation in Hawaii for abdominal pain and hematuria revealed a kidney stone - lithotripsy at Massachusetts General Hospital, Boston, Massachusetts resulted in complete resolution of her pain. In August 1995, she developed diarrhea. Upper gastrointestinal endoscopy with gastric and small intestinal biopsies, as well as colonoscopy and biopsies, were normal. Her symptoms subsequently resolved with no medications.

\section{DISCUSSION}

In a recent multiauthor report from Los Angeles (4), a series of 69 consecutive patients with Crohn's disease were evaluated for serum ANCA. Of these, 18 patients with p-ANCA and clinical features of left-sided colonic inflammatory disease (ie, rectal bleeding, urgency and topical therapies) or ulcerative colitis-like disease were described along with endoscopic and histological documentation of left-sided colitis. The investigators claimed that $100 \%$ of patients with Crohn's disease and this serological marker had ulcerative colitis-like features, indicating that a specific phenotypically distinct group of Crohn's disease could be defined by their p-ANCA status. Moreover, no seropositive patient in their series had small intestinal disease. In contrast to this report from Los Angeles, however, a subsequent study (7) from northern France could not confirm either these ulcerative colitis-like features in p-ANCA-positive patients with Crohn's disease or the direct 'disease-locale' relationship to colonic but not 'isolated' small bowel disease.

Because patient numbers evaluated in Los Angeles (4) were limited, ie, 18 of 69 with ANCA-positive Crohn's disease, it is conceivable that a larger population of patients would have to be examined to confirm the potentially important diagnostic and therapeutic implications of these investigations. To some degree, this concern was addressed by the French study (7), in that 26 of 371 (7\%) patients with Crohn's disease were positive for atypical p-ANCA. In that report, however, the clinical, endoscopic and histological features of these 26 ANCA-positive patients did not differ from those of 26 ANCA-negative patients. It was suggested that these contrasting results in the two investigations were possibly due to inherent differences in the patient populations of Los Angeles and northern France or, alternatively, there were some specific methodological differences in the ANCA assays.

The present study on Canadian patients extended these earlier studies by further evaluating clinical and pathological features in 33 seropositive patients from 247 consecutively evaluated patients with Crohn's disease. A similar patient population was compared with the Los Angeles study (4) with respect to patient age and sex, duration of disease after diagnosis, clinical expression with fistulizing or perforating disease, complications including perianal disease, and therapies including prior steroids or surgery. Moreover, the rates of atypical p-ANCA detection in patients with ulcerative colitis or Crohn's disease using fresh serum samples (3) were similar to those reported from these other centres $(4,7)$. If clinical and endoscopic features (4) alone were considered, an ulcerative colitis-like phenotypic expression was evident in the present study in some, but not all, seropositive patients with Crohn's disease. In the Los Angeles report (4), Crohn's disease was defined by the presence of two or more standard clinical, endoscopic, radiographical and histopathological criteria, including detection of granulomas. In contrast, the investigators indicated that histological definition of an ulcerative colitis-like state in Crohn's disease depended upon failure to detect granulomas. In the present investigation, 29 of 33 patients had granulomas and/or multinucleated giant cells in surgical specimens or serially sectioned endoscopic mucosal biopsies. Thus, some of the differences in the present study may simply reflect the tendency of the Los Angeles group to rely on pathological reports rather than a direct evaluation of histological materials.

Data in the present study also contrast with the results of the Los Angeles study related to disease localization in the small bowel. Small intestinal involvement without colonic involvement was observed in the four seropositive patients in this study that were described in the case descriptions. Although it is conceivable that focal involvement in macroscopically normal colon may have been missed in spite of numerous colonoscopic biopsies from multiple sites within the large intestine, the absence of patients with small bowel disease alone in the Los Angeles study likely reflected the smaller study population. Moreover, the present data are consistent with observations from Northern France (7) that assessed 371 patients with Crohn's disease. In the French study as well as in the present report, small intestinal involvement was present without evident colonic involvement in seropositive patients with Crohn's disease.

Thus, the observations in the present study indicate that patients with Crohn's disease may have different patterns of clinical and pathological disease, and these patterns seem to be independent of their $\mathrm{p}$-ANCA status. This study provides further evidence that both seropositive and seronegative Crohn's disease are heterogeneous inflammatory disease processes.

\section{REFERENCES}

1. Saxon A, Shanahan F, Landers C, Ganz T, Targan S. A distinct subset of antineutrophil cytoplasmic antibodies is associated with inflammatory bowel disease. J Allergy Clin Immunol 1990;86:202-10.

2. Duerr RH, Targan SR, Landers CJ, Sutherland LR, Shanahan F. Neutrophil autoantibodies in ulcerative colitis. Comparison with other colitides/diarrheal diseases. Gastroenterology 1991;100:1590-6.

3. Freeman HJ, Roeck B, Devine D, Carter C. Prospective evaluation of neutrophil autoantibodies in 500 consecutive patients with inflammatory bowel disease. Can J Gastroenterol 1997;11:203-7.

4. Vasiliauskas EA, Plevy SE, Landers CJ, et al. Perinuclear antineutrophil cytoplasmic antibodies in patients with Crohn's disease define a clinical subgroup. Gastroenterology 1996;110:1810-9.

5. Freeman HJ, Roeck B, Devine DV, Carter CJ. Atypical perinuclear antineutrophil cytoplasmic antibodies after colectomy in inflammatory bowel disease. Can J Gastroenterol 1997;11:305-10.

6. Sukhabote J, Freeman HJ. Granulomatous (Crohn's) disease of the upper gastrointestinal tract. A study of 22 patients with mucosal granulomas. Can J Gastroenterol 1993;7:605-9.

7. Jamar-Leclerc N, Reumaux D, Duthilleul P, Colombel JF. Do pANCA define a clinical subgroup in patients with Crohn's disease? Gastroenterology 1997;112:316. 


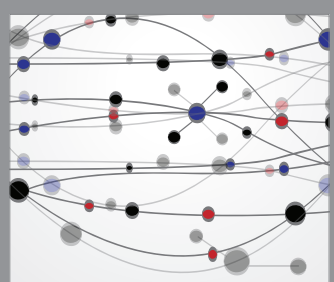

The Scientific World Journal
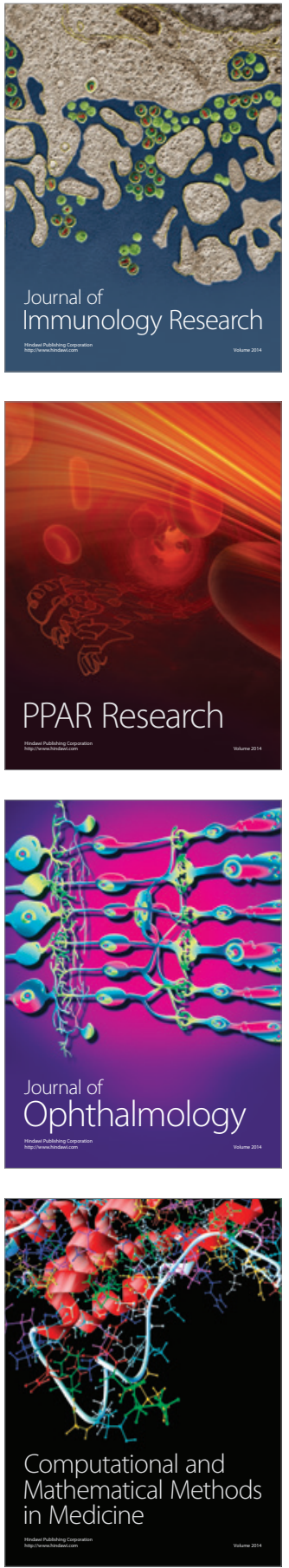

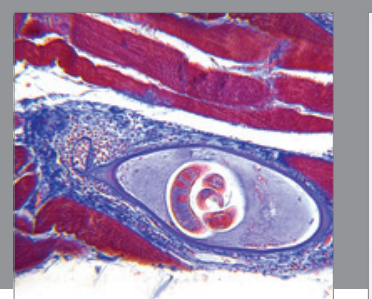

Gastroenterology Research and Practice

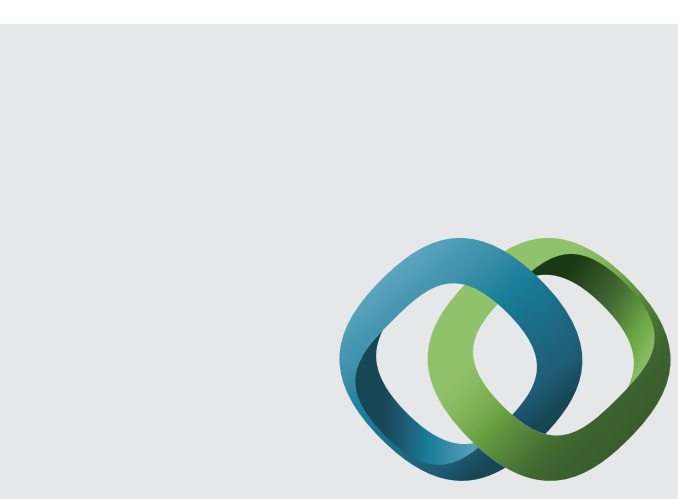

\section{Hindawi}

Submit your manuscripts at

http://www.hindawi.com
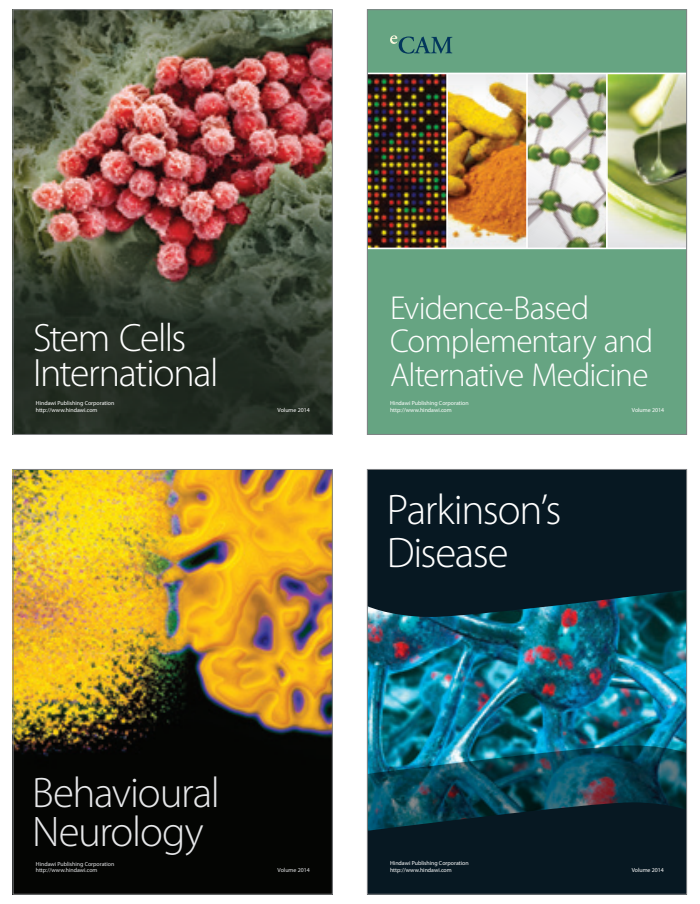
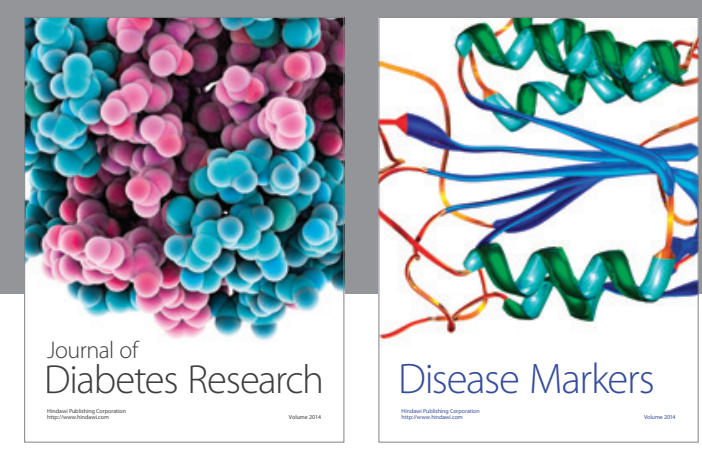

Disease Markers
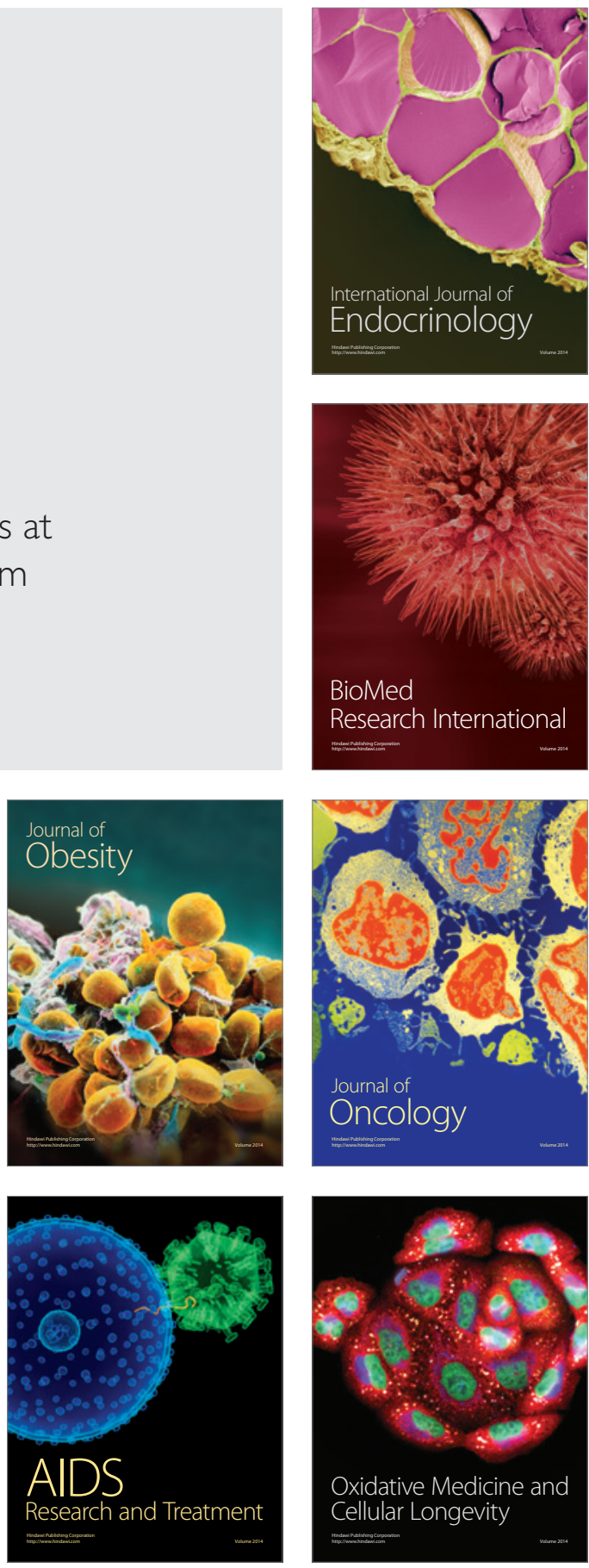